\title{
Structure and function of complex I in animals and plants - A comparative view
}

\author{
Jennifer Senkler ${ }^{1}$, Michael Senkler ${ }^{1}$ and Hans-Peter Braun ${ }^{1}$
}

${ }^{1}$ Institut für Pflanzengenetik, Leibniz Universität Hannover, Herrenhäuser Str. 2, 30419 Hannover, Germany

\section{Corresponding author:}

Hans-Peter Braun, Institute of Plant Genetics, Leibniz Universität Hannover, Herrenhäuser Str. 2, 30419 Hannover, Germany, Tel.: +49 511 7622674, Fax: +49 511 76214351, mail: braun@genetik.uni-hannover.de

$\underline{\text { Running title: }}$

Structure and function of plant complex I

\section{Keywords:}

mitochondria, respiratory chain, NADH ubiquinone oxidoreductase complex, complex I, carbonic anhydrase, Arabidopsis thaliana 


\section{Abstract}

The mitochondrial NADH dehydrogenase complex (complex I) has a molecular mass of about $1000 \mathrm{kDa}$ and includes 40-50 subunits in animals, fungi and plants. It is composed of a membrane arm and a peripheral arm and has a conserved L-like shape in all species investigated. However, in plants and possibly some protists it has a second peripheral domain which is attached to the membrane arm on its matrix exposed side at a central position. The extra domain includes proteins resembling prokaryotic gamma-type carbonic anhydrases. We here present a detailed comparison of complex I from mammals and flowering plants. Forty homologous subunits are present in complex I of both groups of species. In addition, five subunits are present in mammalian complex I, which are absent in plants, and eight to nine subunits are present in plant complex I which do not occur in mammals. Based on the atomic structure of mammalian complex I and biochemical insights into complex I architecture from plants we mapped the species-specific subunits. Interestingly, four of the five animal-specific and five of the eight to nine plant-specific subunits are localized at the inner surface of the membrane arm of complex I in close proximity. We propose that the inner surface of the membrane arm represents a workbench for attaching proteins to complex I not directly related to respiratory electron transport, like nucleoside kinases, acyl-carrier proteins or carbonic anhydrases. We speculate that further enzyme activities might be bound to this micro-location in other groups of organisms.

\section{Abbreviations:}

VCA, $\gamma$-type carbonic anhydrase; $\gamma \mathrm{CAL}, \nu$-type carbonic anhydrase like; $\mathrm{CCM}, \mathrm{CO}_{2}$ concentrating mechanism; ETC: electron transfer chain, EM: electron microscopy; complex I, NADH dehydrogenase complex 
$\underline{\text { Introduction }}$

The mitochondrial NADH dehydrogenase complex (complex I) was first described more than 50 years ago (Hatefi et al. 1962) and represents the first segment of the mitochondrial electron transport chain (ETC) (Hirst 2013). In most organism and at most physiological conditions it represents the main electron entrance point of the ETC. At the same time, it contributes substantially to the formation of the proton gradient across the inner mitochondrial membrane which is used for ATP synthesis by the ATP synthase complex. Complex $I$ is composed of two elongated domains designated the "membrane arm", which is mainly embedded into the inner mitochondrial membrane, and the "peripheral arm", which protrudes into the mitochondrial matrix. The two arms are connected end-by-end forming an L-like particle (Hofhaus et al. 1991, Weiss et al. 1991). NADH oxidation takes place at the tip of the peripheral arm. Within the peripheral arm, electrons are transported via a series of ironsulfur clusters to ubiquinone (see Hirst and Roessler 2016 for review). Ubiquinone reduction causes conformational changes of the complex I particle, which drive translocation of protons from the mitochondrial matrix to the mitochondrial intermembrane space through the membrane arm (reviewed in Wirth et al. 2016). Overall, four protons are translocated per oxidized NADH.

Insights into complex I structure and function initially came from prokaryotic complex I. This complex is much smaller than mitochondrial complex I, but basically fulfills the same function in respiratory electron transport. Complex I from E. coli includes 14 subunits, seven of which are localized in the peripheral arm and seven of which form part of the membrane arm. These fourteen proteins are called the 'minimal-set' or 'core' subunits. The overall mass of $E$. coli complex I is around 550 kDa (Leif et al. 1995, Berrisford et al. 2016). Some bacteria include one to three further accessory subunits and are slightly larger. Complex I from Thermus thermophilus was recently characterized by X-ray crystallography at atomic resolution (Baradaran et al. 2013, Berrisford et al. 2016).

Compared to prokaryotic complex I, mitochondrial complex comprises 25-35 extra subunits in mammals, fungi and plants (Cardol 2011, Hirst 2013, Braun et al. 2014, Wirth et al. 2016). The functions of most of these subunits are currently not known. The overall molecular mass of 
eukaryotic complex I is around $1000 \mathrm{kDa}$. In 2016, structures of mammalian complex I were characterized at atomic resolution by single particle cryo electron microscopy (Zhu et al. 2016, Fiedorczuk et al. 2016). Mammalian complex I includes 45 proteins (43 single copy subunits; one protein [the SDAP subunit] present in two copies). Based on the crystal structures the extra subunits are seen to form some kind of cage around the 'minimal-set' subunits, thereby probably stabilizing the respiratory complex. This cage might be necessary to compensate for comparatively lower hydrophobicity of the 'minimal-set'-subunits in eukaryotes (this is interpreted to be a consequence of mitochondrial evolution after initial endosymbiosis, which was accompanied by massive gene transfer from the mitochondria to the cell nucleus and the development of protein import pathways into the organelle, van der Sluis et al. 2015).

\section{Mitochondrial complex I from plants}

Mitochondrial complex I of plants has not been analyzed with atomic resolution. In addition to complex I, plant mitochondria include some "alternative" NADH dehydrogenases and also an alternative oxidase (reviewed by Millar et al. 2011). As a result, the electron transfer system of plants is highly branched. The alternative enzymes participate in respiratory electron transport without contributing to the proton gradient across the inner mitochondrial membrane and are believed to be important in the context of the plant stress response. They allow stabilizing the redox balance of plant cells, e.g. at high-light conditions. Their presence has implications for the physiological role of complex I. However, also in plants, complex I is an essential site for electron flow into the ETC under most physiological conditions, as deletion of complex I genes causes drastic delay in plant development and loss of plant fertility (Fromm et al. 2016a).

A first low-resolution structure of Arabidopsis complex I obtained by single particle EM revealed that the enzyme has a unique shape (Fig. 1a, Dudkina et al. 2005). The L-like structure is conserved, but it includes an additional spherical domain which is attached to the membrane arm on its matrix-exposed side at a central position. At the same time, systematic analyses of complex I subunits from Arabidopsis revealed the presence of proteins unique to plants, e.g. a group of five structurally related proteins resembling prokaryotic $\gamma$-type carbonic anhydrases (Heazlewood et al. 2003, Parisi et al. 2004, Perales et al. 2004, Braun et al. 2014). 
Indeed it has been shown that the carbonic anhydrase subunits of plant complex I are included in the extra spherical domain (Sunderhaus et al. 2006), which is therefore called the carbonic anhydrase domain (Fromm et al. 2016b). This domain is conserved in potato (Bultema et al. 2009, Fig. 1b) and maize (Peters et al. 2008) and also in the alga Polytomella, which is related to Chlamydomonas (Sunderhaus et al. 2006). Genes encoding the complex I-associated carbonic anhydrase subunits are present in the genomes of higher plants, alga as well as some protists (Heazlewood et al. 2003, Cardol et al. 2004, Gawryluk and Gray 2010, Braun et al. 2014, Subrahmanian et al. 2016).

Compared to prokaryotic $\gamma$-type carbonic anhydrases, three of the five complex I-associated carbonic anhydrase subunits of Arabidopsis have conserved active sites (Parisi et al. 2004, Perales et al. 2004). These are termed Carbonic Anhydrase subunits 1, 2 and 3 (CA1, CA2 and CA3). Two further subunits lack some amino acids involved in zinc binding and probably have no carbonic anhydrase activity. They were suggested to be called Carbonic $\underline{\text { Anhydrase-Like }}$ subunits 1 and 2 (CAL1 and CAL2) (Perales et al. 2004). The carbonic anhydrase domain has a mass of about $85 \mathrm{kDa}$ and probably includes two CA-subunits and one CAL subunit (reviewed in Fromm et al. 2016b). CAL1 and CAL2 are highly similar and most likely represent isoforms; CA3 seems to be less abundant than CA1 and CA2 and might substitute for one of these proteins under currently unknown conditions.

It has been speculated that the complex I associated carbonic anhydrases may be involved in a mechanism allowing transfer of mitochondrial $\mathrm{CO}_{2}$ to the chloroplasts for re-fixation by the Calvin cycle (Braun and Zabaleta 2007, Zabaleta et al. 2012). This mechanism is somehow analogous to the well-studied carbon concentrating mechanism (CCM) of cyanobacteria, which also depends on cyanobacterial complex I (Kupriyanova et al. 2013). It is based on the conversion of $\mathrm{CO}_{2}$ into bicarbonate in the mitochondria, bicarbonate export to the cytoplasm, its import into chloroplasts and finally reconversion of bicarbonate into $\mathrm{CO}_{2}$ in the chloroplast stroma (Zabaleta et al. 2012). If true, knock-out mutants with respect to the complex Iintegrated carbonic anhydrase subunits might be rescued if plants are cultivated at elevated $\mathrm{CO}_{2}$, as has been reported for mutants with respect to genes involved in photorespiration (Timm and Bauwe 2013). 
Recently, several single and double knock-out mutants with respect to the complex Iassociated carbonic anhydrases have been characterized in Arabidopsis thaliana (summarized in Table 1). Surprisingly, deletion of single genes encoding CA or CAL subunits did not cause any visible phenotypes under all of the conditions tested (Perales et al. 2005, Wang et al. 2012). However, gene deletions clearly affected complex I assembly, especially if the gene encoding CA2 was deleted (Perales et al. 2005). The CA/CAL proteins are important for early steps in complex I assembly as revealed by analyses involving complex I mutants and by pulsechase experiments using isotopes (Meyer et al. 2011, Li et al. 2013). Simultaneous deletion of two of the genes encoding CA/CAL proteins caused strong developmental problems at the seed stage (CAL1+CAL2 deletion or CA1+CA2 deletion), except if one of the two deleted genes encoded CA3 (e.g. CA1+CA3 deletion) (Wang et al. 2012, Cordoba et al. 2016, Fromm et al. 2016a, Fromm et al. 2016d). Seeds from CA1/CA2 double-mutant could be rescued by cultivation on sucrose; plants could be regenerated, but were strongly delayed in development (Fromm et al. 2016d). Complex I was completely absent in the CA1/CA2 double mutant. If the CA1/CA2 double mutant was transformed with a $\mathrm{Ca} 2$ gene encoding an altered version of CA2 defective in the carbonic anhydrase active site, complex I assembly was restored to normal levels (Fromm et al. 2016d). At a phenotype level, the transformants resembled wild-type plants. The postulated $\mathrm{CO}_{2}$ transfer mechanism was not supported by these observations, but also not excluded. In contrast, a mutant lacking CA2 and additionally one of the two genes encoding the CAL subunits exhibited a reduced growth rate, which was partially rescued by cultivation at elevated $\mathrm{CO}_{2}$ (Soto et al. 2015). In summary, the physiological role of the complex l-associated carbonic anhydrases is not entirely clear.

\section{Comparison of plant and animal complex I}

The recently determined atomic structures of complex I from Bos taurus and Ovis aries (Zhu et al. 2016, Fiedorczuk et al. 2016) allow drawing new conclusions on the structure and function of plant complex I. We here present a detailed comparison of complex I from flowering plants and mammals. The complex I structures from Bos taurus and Ovis aries include 45 subunits. Analysis of complex I subunits from Arabidopsis revealed 49 subunits (Peters et al. 2013, Braun et al. 2014). Overall, 40 homologous subunits are present in complex I from Bos taurus, Ovis aries and Arabidopsis (the 14 'minimal set' subunits and 26 of the 
accessory subunits, Table 2). Five proteins are present in the mammalian complex I, but absent in Arabidopsis (the $42 \mathrm{kDa}, \mathrm{B} 17, \mathrm{~B} 10, \mathrm{SDAP}-\alpha, \mathrm{SDAP}-\beta$ subunits). On the other hand, eight to nine subunits are present in Arabidopsis complex I, but absent in mammals. These are the five CA/CAL subunits, two small proteins called P1 and P2 and another protein, which resembles subunits of the pre-protein translocase of the inner mitochondrial membrane ("tim-like" subunit). Finally, the L-galactono-1,4-lactone dehydrogenase (GLDH), which catalyzes the terminal step of ascorbate biosynthesis, is associated with complex I in plants (Heazlewood et al. 2003, Millar et al. 2004). This protein probably only binds to subcomplexes of complex I and has been shown to be essential for complex I assembly in Arabidopsis (Pineau et al. 2008, Schertl et al. 2012, Schimmeyer et al. 2016). However, its binding to intact complex I cannot be excluded because it also has been detected by mass spectrometry in a fraction containing the purified holo complex (Peters et al. 2013).

Some further plant-specific proteins are discussed to be candidates for additional complex I subunits (Peters et al. 2013, Braun et al. 2014). However, their association with complex I could not be confirmed by a recent complexome profiling analysis using a total mitochondrial fraction of Arabidopsis thaliana (Senkler et al 2017). These proteins therefore are not included in table 2.

In an attempt to obtain further insights into the structure of plant complex I, we subtracted subunits of the complex I structure of Bos taurus which are absent in Arabidopsis (Fig. 2). Interestingly, four of the five subunits ( $42 \mathrm{kDa}, \mathrm{B} 17, \mathrm{SDAP}-\alpha, \mathrm{SDAP}-\beta$ ) are localized at the inner surface of the membrane arm at a region, which is required for anchoring the carbonic anhydrase domain in plants. Only the $10 \mathrm{kDa}$ subunit absent in Arabidopsis is localized at a different location, the tip of the peripheral arm. Five further subunits of Arabidopsis complex I (the ASHI, B22, B12, B15 and SGDH subunits), which also are localized the inner surface of the membrane arm of complex I, lack sequence stretches if compared to the corresponding bovine sequences (see Appendix S1). This further contributes to a clear reduction in mass at the site required for the anchoring of the carbonic anhydrase domain (Fig. 2).

We finally compared the complex I structure of Bos taurus lacking the five subunits absent in Arabidopsis thaliana and the $\mathrm{N}$-terminal or $\mathrm{C}$-terminal portions of the five additional subunits 
with the low-resolution EM structures of complex I from Arabidopsis and potato (Fig. 3). Interestingly, the reduced complex I from Bos taurus strikingly resembles complex I from Arabidopsis and potato. Clearly, the carbonic anhydrase domain is a unique feature of complex I in plants. Furthermore, the overall length of the membrane arm of plant complex I is increased (already described by Dudkina et al. 2005), indicating that the additional plantspecific subunits possibly are located at the tip or an interior position of the membrane arm.

Investigation of complex I assembly in Arabidopsis revealed that CA/CAL proteins form an 200 kDa assembly intermediate together with the "20.9 kDa" subunit (Li et al. 2013) which also is called the MNLL protein (see table 2). Indeed, position of this subunit within the membrane arm of bovine complex $I$ is exactly at the site of attachment of the carbonic anhydrase domain to the membrane arm within plant complex I. Analyses of a high-resolution structure of complex I from Arabidopsis will be necessary to obtain more detailed information on the precise anchoring of the CA/CAL domain into complex I in plants.

The inner surface of the membrane arm of complex I represents a workbench for attaching enzyme activities

It has been suggested that enzymes unrelated to complex I function have been anchored to the complex during evolution of distinct eukaryotic lineages (Cardol 2011). Interestingly, these enzymes activities seem to be preferentially bound to the matrix-exposed surface of the membrane arm of complex I. The $42 \mathrm{kDa}$ subunit of mammals, which is absent in plants, belongs to the nucleoside kinase family. The SDAP- $\alpha / \beta$ subunits, also absent in plant complex I (Meyer et al. 2007), are acyl-carrier proteins and involved in fatty acid biosynthesis (Zensen et al. 1992). Finally, the carbonic anhydrase subunits absent in mammals are attached to complex I from plants at the corresponding position (Sunderhaus et al. 2006). The inner surface of the membrane arm of complex $I$ is an extraordinarily large protein surface within the inner mitochondrial membrane, which seems to be an ideal workbench for anchoring additional enzyme activities, which might benefit from membrane location. Furthermore, the inner surface of the membrane arm is a very basic micro-location, which promotes $\mathrm{pH}$ dependent reactions. For instance, the reaction equilibrium of the $\mathrm{CO}_{2} /$ bicarbonate conversion is strongly on the bicarbonate side at basic $\mathrm{pH}$. It will be interesting to see complex 
I structures from other groups of organisms with respect to the inner surface of the membrane arm. Overall, complex I structure in Eukaryotes is remarkably conserved, but we predict structural heterogeneity especially at this site.

\section{Author contributions}

JS analyzed data, MS analyzed data and prepared figures, HPB analyzed data, prepared figures and wrote the paper.

\section{Acknowledgements}

This work was supported by the Deutsche Forschungsgemeinschaft (DFG), Forschergruppe 1186 (grant Br1829/10-2). 


\section{$\underline{\text { References }}$}

Baradaran R, Berrisford JM, Minhas GS, Sazanov LA (2013) Crystal structure of the entire respiratory complex I. Nature 494: 443-48

Berrisford JM, Baradaran R, Sazanov LA (2016) Structure of bacterial respiratory complex I. Biochim Biophys Acta 1857: 892-901

Braun HP, Zabaleta E (2007) Carbonic anhydrase subunits of the mitochondrial NADH dehydrogenase complex (complex I) in plants. Physiol Plant 129: 114-122

Braun HP, Binder S, Brennicke A, Eubel H, Fernie AR, Finkemeier I, Klodmann J, König AC, Kühn K, Meyer EH, Obata T, Schwarzländer M, Takenaka M, Zehrmann A (2014) The life of plant mitochondrial complex I. Mitochondrion 19 (Pt B): 295-313

Bultema J, Braun HP, Boekema E, Kouril R (2009) Megacomplex organization of the oxidative phosphorylation system by structural analysis of respiratory supercomplexes frompotato. Biochim Biophys Acta 1787: 60-67

Cardol P (2011) Mitochondrial NADH:ubiquinone oxidoreductase (complex I) in eukaryotes: a highly conserved subunit composition highlighted by mining of protein databases. Biochim Biophys Acta 1807: $1390-1397$

Cardol P, Vanrobaeys F, Devreese B, Van Beeumen J, Matagne RF, Remacle C. (2004) Higher plant-like subunit composition of mitochondrial complex I from Chlamydomonas reinhardtii: 31 conserved components among eukaryotes. Biochim Biophys Acta 1658: 212-224

Córdoba JP, Marchetti F, Soto D, Martin MV, Pagnussat GC, Zabaleta E (2016) The CA domain of the respiratory complex I is required for normal embryogenesis in Arabidopsis thaliana. J Exp Bot 67: 15891603

Dudkina NV, Eubel H, Keegstra W, Boekema EJ, Braun HP (2005) Structure of a mitochondrial supercomplex formed by respiratory-chain complexes I and III. Proc Natl Acad Sci U.S.A 102: 32253229 
Fiedorczuk K, Letts JA, Degliesposti G, Kaszuba K, Skehel M, Sazanov LA (2016) Atomic structure of the entire mammalian mitochondrial complex I. Nature 538: 406-410

Fromm S, Senkler J, Eubel H, Peterhänsel C, Braun HP (2016a) Life without complex I: Proteome analyses of an Arabidopsis mutant lacking the mitochondrial NADH dehydrogenase complex. J Exp Bot 67: 3079-3093

Fromm S, Senkler J, Peterhänsel C, Braun HP (2016b) The carbonic anhydrase domain of plant mitochondrial complex I. Physiologia Plantarum 157, 289-296

Fromm S, Göing J, Lorenz C, Peterhänsel C, Braun HP (2016c) Depletion of the "gamma-type carbonic anhydrase-like" subunits of complex I affects central mitochondrial metabolism in Arabidopsis thaliana. Biochim Biophys Acta 1857: 60-71

Fromm S, Braun HP, Peterhänsel C (2016d) Mitochondrial gamma carbonic anhydrases are required for complex I assembly and plant reproductive development. New Phytol 211: 194-207

Gawryluk RM, Gray MW (2010) Evidence for an early evolutionary emergence of gamma-type carbonic anhydrases as components of mitochondrial respiratory complex I. BMC Evol Biol 10: 176

Heazlewood JL, Howell KA, Millar AH (2003) Mitochondrial complex I from Arabidopsis and rice: orthologs of mammalian and fungal components coupled with plant-specific subunits. Biochim Biophys Acta 1604: 159-169

Hatefi Y, Haavik AG, Griffiths DE (1962) Studies on the electron transfer system XL: preparation and properties of mitochondrial DPNH-coenzyme Q reductase. J Biol Chem 237: 1676-1680

Hirst J (2013) Mitochondrial complex I. Annu Rev Biochem 82: 551-575

Hirst J, Roessler, MM (2016) Energy conversion, redox catalysis and generation of reactive oxygen species by respiratory complex I. Biochim Biophys Acta 1857: 872-883

Hofhaus G, Weiss H, Leonard K (1991) Electron microscopic analysis of the peripheral and membrane parts of mitochondrial NADH dehydrogenase (complex I). J Mol Biol 221: 1027-1043 
Kupriyanova EV, Sinetova MA, Cho SM, Park YI, Los DA, Pronina NA (2013) $\mathrm{CO}_{2}$-concentrating mechanism in cyanobacterial photosynthesis: organization, physiological role, and evolutionary origin. Photosynth Res 117, 133-146

Leif H, Sled VD, Ohnishi T, Weiss H, Friedrich, T (1995) Isolation and characterization of the protontranslocating NADH: ubiquinone oxidoreductase from Escherichia coli. Eur J Biochem 230: 538-548

Li L, Nelson CJ, Carrie C, Gawryluk RM, Solheim C, Gray MW, Whelan J, Millar AH (2013) Subcomplexes of ancestral respiratory complex I subunits rapidly turn over in vivo as productive assembly intermediates in Arabidopsis. J Biol Chem 288: 5707-5717

Meyer EH, Heazlewood JL, Millar AH (2007) Mitochondrial acyl carrier proteins in Arabidopsis thaliana are predominantly soluble matrix proteins and none can be confirmed as subunits of respiratory complex I. Plant Mol Biol 64: 319-327

Meyer EH, Solheim C, Tanz SK, Bonnard G, Millar AH (2011) Insights into the composition and assembly of the membrane arm of plant complex I through analysis of subcomplexes in Arabidopsis mutant lines. J Biol Chem 286: 26081-26092

Millar AH, Mittova V, Kiddle G, Heazlewood JL, Bartoli CG, Theodoulou FL, Foyer CH (2003) Control of ascorbate synthesis by respiration and its implications for stress responses. Plant Physiol 133: 443-447

Millar AH, Whelan J, Soole KL, Day DA (2011) Organization and regulation of mitochondrial respiration in plants. Annu Rev Plant Biol 62: 79-104

Parisi G, Perales M, Fornasari MS, Colaneri A, Gonzalez-Schain N, Gomez-Casati D, Zimmermann S, Brennicke A, Araya A, Ferry JG, Echave J, Zabaleta E (2004) Gamma carbonic anhydrases in plant mitochondria. Plant Mol Biol 55: 193-207

Perales M, Parisi G, Fornasari MS, Colaneri A, Villarreal F, Gonzalez-Schain N, Echave J, Gomez-Casati D, Braun HP, Araya A, Zabaleta E (2004) Gamma carbonic anhydrase like complex interact with plant mitochondrial complex I. Plant Mol Biol 56: 947-957 
Perales M, Eubel H, Heinemeyer J, Colaneri A, Zabaleta E, Braun HP (2005) Disruption of a nuclear gene encoding a mitochondrial gamma carbonic anhydrase reduces complex I and supercomplex I+III2 levels and alters mitochondrial physiology in Arabidopsis. J Mol Biol 350: 263-277

Peters K, Dudkina NV, Jänsch L, Braun HP, Boekema EJ (2008) A structural investigation of complex I and $\mathrm{I}+\mathrm{III} \mathrm{I}_{2}$ supercomplex from Zea mays at $11-13$ A resolution: assignment of the carbonic anhydrase domain and evidence for structural heterogeneity within complex I. Biochim Biophys Acta 1777: 84-93

Peters K, Belt K, Braun HP (2013) 3D Gel Map of Arabidopsis Complex I. Front Plant Sci 4: 153

Pineau B, Layoune O, Danon A, De Paepe R (2008) L-Galactono-1,4-lactone dehydrogenase is required for the accumulation of plant respiratory complex I. J Biol Chem 283: 32500-32505

Schertl P, Sunderhaus S, Klodmann J, Grozeff GE, Bartoli CG, Braun HP (2012) L-galactono-1,4-lactone dehydrogenase (GLDH) forms part of three subcomplexes of mitochondrial complex I in Arabidopsis thaliana. J Biol Chem 287: 14412-14419

Schimmeyer J, Bock R, Meyer EH (2016) L-Galactono-1,4-lactone dehydrogenase is an assembly factor of the membrane arm of mitochondrial complex I in Arabidopsis. Plant Mol Biol 90: 117-126

Senkler J, Senkler M, Eubel H, Hildebrandt T, Lengwenus C, Schertl P, Schwarzländer M, Wagner S, Wittig I, Braun, HP (2017) The mitochondrial complexome of Arabidopsis thaliana. Plant J., in press, doi: $10.1111 /$ tpj.13448

Soto D, Córdoba JP, Villarreal F, Bartoli C, Schmitz J, Maurino V, Braun HP, Pagnussat GC, Zabaleta E (2015) Functional characterization of mutants affected in the carbonic anhydrase domain of the respiratory complex I in Arabidopsis thaliana. Plant J 83: 831-844

Subrahmanian N, Remacle C, Hamel PP (2016) Plant mitochondrial Complex I composition and assembly: A review. Biochim Biophys Acta 1857, 1001-1014

Sunderhaus S, Dudkina N, Jänsch L, Klodmann J, Heinemeyer J, Perales M, Zabaleta E, Boekema E, Braun HP (2006) Carbonic anhydrase subunits form a matrix exposed domain attached to the membrane arm of mitochondrial complex I in plants. J Biol Chem 281: 6482-6488 
Timm S, Bauwe H. (2013) The variety of photorespiratory phenotypes - employing the current status for future research directions on photorespiration. Plant Biol (Stuttgart) 15: 737-747

van der Sluis EO, Bauerschmitt H, Becker T, Mielke T, Frauenfeld J, Berninghausen O, Neupert W, Herrmann JM, Beckmann R (2015) Parallel Structural Evolution of Mitochondrial Ribosomes and OXPHOS Complexes. Genome Biol Evol 7: 1235-1251

Villarreal F, Martín V, Colaneri A, González-Schain N, Perales M, Martín M, Lombardo C, Braun HP, Bartoli C, Zabaleta E. (2009) Ectopic expression of mitochondrial gamma carbonic anhydrase 2 causes male sterility by anther indehiscence. Plant Mol Biol 70: 471-485

Wang Q, Fristedt R, Yu X, Chen Z, Liu H, Lee Y, Guo H, Merchant SS, Lin C (2012) The Y-carbonic anhydrase subcomplex of mitochondrial complex $I$ is essential for development and important for photomorphogenesis of Arabidopsis. Plant Physiol 160: 1373-1383

Weiss H, Friedrich T, Hofhaus G, Preis D (1991) The respiratory-chain NADH dehydrogenase (complex I) of mitochondria. Eur J Biochem 197: 563-576

Wirth C, Brandt U, Hunte C, Zickermann V (2016) Structure and function of mitochondrial complex I. Biochim Biophys Acta 1857: 902-914

Zabaleta E, Martin MV, Braun HP (2012) A basal carbon concentrating mechanism in plants? Plant Sci 187: $97-104$

Zensen R, Husmann H, Schneider R, Peine T, Weiss H (1992) De novo synthesis and desaturation of fatty acids at the mitochondrial acyl-carrier protein, a subunit of NADH:ubiquinone oxidoreductase in Neurospora crassa. FEBS Lett 310: 179-181

Zhu J, Vinothkumar KR, Hirst J (2016) Structure of mammalian respiratory complex I. Nature 536: 354-358 


\section{Supporting Information}

Appendix S1:

Determination of the mature N-termini of complex I subunits from Arabidopsis thaliana

Appendix S2:

Pairwise alignments of homologous complex I subunits from Arabidopsis thaliana and Bos taurus 


\section{Tables}

Table 1: Complex I mutants affected in carbonic anhydrase subunits in Arabidopsis thaliana

\begin{tabular}{|c|c|c|c|}
\hline \multicolumn{2}{|c|}{ mutation(s) } & \multirow[t]{2}{*}{ observations } & \multirow[t]{2}{*}{ reference } \\
\hline $1^{\text {st }}$ & $2^{\text {nd }}$ & & \\
\hline$\triangle$-CA2 & - & $\begin{array}{l}\text { - no visible phenotype under standard growth conditions } \\
\text { - reduced growth rate / reduced respiration rate of a } \\
\text { suspension cell culture } \\
\text { - complex I reduced by } 50-80 \% \\
\text { - absence of CA2 in the carbonic anhydrase domain is } \\
\text { compensated by other CA/CALs } \\
\text { - CA2 is incorporated into complex I at an early stage during } \\
\text { complex I assembly }\end{array}$ & $\begin{array}{l}\text { Perales et al. } 2005, \\
\text { Wang et al. } 2012 \\
\text { Sunderhaus et al. } 2006 \\
\text { Meyer et al. } 2011\end{array}$ \\
\hline$\Delta$-CA1 & - & - no visible phenotype under standard growth conditions & Wang et al. 2012 \\
\hline$\triangle-\mathrm{CA} 3$ & - & - no visible phenotype under standard growth conditions & $\begin{array}{l}\text { Perales et al. } 2005 \\
\text { Wang et al. } 2012\end{array}$ \\
\hline$\Delta-\mathrm{CAL1}$ & - & - no visible phenotype under standard growth conditions & Wang et al. 2012 \\
\hline$\triangle-C A L 2$ & - & $\begin{array}{l}\text { - no visible phenotype under standard growth conditions } \\
\text { - no reduced complex I levels }\end{array}$ & $\begin{array}{l}\text { Wang et al. } 2012 \\
\text { Meyer et al. } 2011\end{array}$ \\
\hline$\Delta$-CA1 & $\triangle$-CA3 & - no visible phenotype under standard growth conditions & Wang et al. 2012 \\
\hline$\Delta$-CAL1 & $\Delta$-CAL2 & - defective embryogenesis and non-germinating seeds & Wang et al. 2012 \\
\hline$\triangle$-CAL1 & i-CAL2 & $\begin{array}{l}\text { - reduced plant growth; altered photomorphogenic } \\
\text { - covelopment } \\
\text { - induction of a stress response reaction } \\
\text { - central mitochondrial metabolism affected }\end{array}$ & $\begin{array}{l}\text { Wang et al. } 2012 \\
\text { Fromm et al. } 2016 \text { c }\end{array}$ \\
\hline$\triangle$-CA2 & $\Delta$-CAL1 & $\begin{array}{l}\text { - } \text { reduced plant growth; partial compensation if plants are } \\
\text { cultivated at elevated } \mathrm{CO}_{2} \text { (mild photorespiratory phenotype) } \\
\text { - increased glycine levels } \\
\text { - increased ROS }\end{array}$ & Soto et al. 2015 \\
\hline$\triangle-C A 2$ & $\triangle-C A L 2$ & $\begin{array}{l}\text { - } \text { reduced plant growth; partial compensation if plants are } \\
\text { cultivated at elevated } \mathrm{CO}_{2} \text { (mild photorespiratory phenotype) } \\
\text { - increased glycine levels } \\
\text { - increased ROS }\end{array}$ & Soto et al. 2015 \\
\hline$\Delta$-CA1 & $\triangle-C A 2$ & $\begin{array}{l}\text { - embryogenesis and seed development strongly disturbed } \\
\text { - drastically reduced plant development } \\
\text { - complex I completely absent } \\
\text { - complexes II and IV upregulated } \\
\text { - alternative respiratory enzymes increased } \\
\text { - glycolysis and citric acid cycle enzymes increased } \\
\text { - enzymes involved in amino acid catabolism increased } \\
\text { - fermentation increased } \\
\text { - photosystems decreased } \\
\text { - increased stress symptoms, increased ROS }\end{array}$ & $\begin{array}{l}\text { Cordoba et al. } 2016 \\
\text { Fromm et al. } 2016 \text { a } \\
\text { Fromm et al. } 2016 \text { d }\end{array}$ \\
\hline $0-\mathrm{CA} 2$ & - & - male sterility & Villarreal et al. 2009 \\
\hline
\end{tabular}

$\Delta=$ homozygous knock out mutant, $\mathrm{i}=$ knock down by RNA interference, $\mathrm{O}=$ overexpression 
Table 2: Complex I subunits in A. thaliana and B. taurus

\begin{tabular}{|c|c|c|c|c|c|}
\hline Subunit $^{1}$ & A. thaliana & B. taurus & kDa A. thaliana ${ }^{2}$ & kDa B. taurus ${ }^{3}$ & seq similarity ${ }^{4}$ \\
\hline \multicolumn{6}{|c|}{ Peripheral arm } \\
\hline 13 kDa & At3g03070 & P23934 & 9.7 & 10.5 & 46.2 \\
\hline $18 \mathrm{kDa}$ & At5g67590 & Q02375 & 14.4 & 15.3 & 55.9 \\
\hline 39 kDa & At2g20360 & P34943 & 37.7 & 39.1 & 55.4 \\
\hline $51 \mathrm{kDa}$ & At5g08530 & P25708 & 50.1 & 48.5 & 82.3 \\
\hline $75 \mathrm{kDa}$ & At5g37510 & P15690 & 75.1 & 77.0 & 69.5 \\
\hline B8 & At5g47890 & Q02370 & 10.7 & 10.9 & 63.4 \\
\hline B13 & At5g52840 & P23935 & 17.7 & 13.2 & 41.5 \\
\hline B14.5a & At5g08060 & Q05752 & 14.9 & 12.5 & 25.2 \\
\hline PSST & At5g11770 & P42026 & 21.6 & 20.1 & 70.5 \\
\hline TYKY & At1g79010, At1g16700 & P42028 & $23.7,23.6$ & 20.2 & 72.5 \\
\hline
\end{tabular}

Membrane arm

\begin{tabular}{|c|c|c|c|c|c|}
\hline $15 \mathrm{kDa}$ & At3g62790, At2g47690 & Q02379 & $9.8,11.4$ & 12.5 & 30.6 \\
\hline 42 kDa & & P34942 & - & 39.3 & - \\
\hline AGGG & At1g76200 & Q02374 & 7.4 & 8.5 & 25.8 \\
\hline ASHI & At5g47570 & Q02372 & 10.2 & 18.7 & 19.0 \\
\hline B9 & At2g46540 & Q02371 & 6.7 & 9.2 & 28.2 \\
\hline B12 & At1g14450, At2g02510 & Q02365 & $8.1,7.9$ & 11.0 & 25.9 \\
\hline B14 & At3g12260 & Q02366 & 15.0 & 14.9 & 47.5 \\
\hline B14.5b & At4g20150 & Q02827 & 9.2 & 14.1 & 21.0 \\
\hline B14.7 & At2g42210 & Q8HXG6 & 17.0 & 14.6 & 30.2 \\
\hline B15 & At2g31490 & P48305 & 8.2 & 15.1 & 24.8 \\
\hline B16.6 & At1g04630, At2g33220 & Q95KV7 & $16.0,16.0$ & 16.5 & 52.2 \\
\hline B17 & & Q02367 & - & 15.4 & - \\
\hline B18 & At2g02050 & Q02368 & 11.7 & 16.3 & 40.3 \\
\hline B22 & At4g34700 & Q02369 & 13.5 & 21.7 & 33.0 \\
\hline ESSS-1 & At2g42310, At3g57785 & Q8HXG5 & $10.1,10.1$ & 14.5 & 29.0 \\
\hline KFYI & At4g00585 & Q02376 & 9.7 & 5.8 & 12.0 \\
\hline MNLL & At4g16450 & Q24JZO & 11.3 & 7.0 & 13.0 \\
\hline MWFE & At3g08610 & Q02377 & 7.2 & 8.1 & 43.1 \\
\hline ND1 & AtMg00516 / AtMg01120 / AtMg01275 & P03887 & 35.7 & 35.7 & 66.7 \\
\hline ND2 & AtMg00285 / AtMg01320 & P03892 & 54.9 & 39.3 & 31.4 \\
\hline ND3 & AtMg00990 & P03898 & 13.7 & 13.1 & 48.8 \\
\hline ND4 & AtMg00580 & P03910 & 55.1 & 52.1 & 48.0 \\
\hline ND4L & AtMg00650 & Q3L5T0 & 10.9 & 10.8 & 45.8 \\
\hline ND5 & AtMg00060 / AtMg00513 / AtMg00665 & P03920 & 73.9 & 68.3 & 46.6 \\
\hline ND6 & AtMg00270 & P03924 & 23.5 & 19.1 & 36.7 \\
\hline PDSW & At1g49140, At3g18410 & Q02373 & $12.3,12.4$ & 20.8 & 21.7 \\
\hline PGIV & At3g06310, At5g18800 & P42029 & $12.2,11.8$ & 20.0 & 29.1 \\
\hline SDAP- $\alpha$ & & P52505 & - & 10.1 & - \\
\hline SDAP- $\beta$ & & P52505 & - & 10.1 & - \\
\hline SGDH & At1g67785 & Q02380 & 7.4 & 16.7 & 19.6 \\
\hline CA1 & At1g19580 & - & 29.8 & - & - \\
\hline CA2 & At1g47260 & - & 29.9 & - & - \\
\hline CA3 & At5g66510 & - & 27.8 & - & - \\
\hline CAL1 & At5g63510 & - & 25.1 & - & - \\
\hline CAL2 & At3g48680 & - & 25.0 & - & - \\
\hline GLDH & At3g47930 & - & 68.6 & - & - \\
\hline P1 & At1g67350 & - & 11.7 & - & - \\
\hline P2 & At2g27730 & - & 11.8 & - & - \\
\hline TIM-like & At1g18320, At3g10110 & - & $15.1,18.1$ & - & - \\
\hline
\end{tabular}

${ }^{1}$ Subunits present in bovine complex I, but absent in complex I from Arabidopsis, are shown in red. Subunits present in Arabidopsis, but absent in bovine complex I, are shown in blue. Subunits at the 
matrix exposed surface of the membrane arm, which lack N-terminal or C-terminal portions in Arabidopsis with respect to the bovine subunits, are indicated in orange.

${ }^{2}$ Molecular masses of the mature subunits. If subunits are encoded as precursors, the presequences are subtracted. See Appendix S1 for details.

${ }^{3}$ Molecular masses of the mature subunits. Data were taken from Zhu et al. 2016, Supp. Material.

${ }^{4}$ Similarity of the mature sequences in B. taurus and A. thaliana given as \% homology, see Appendix S2 for details. 


\section{Figures}
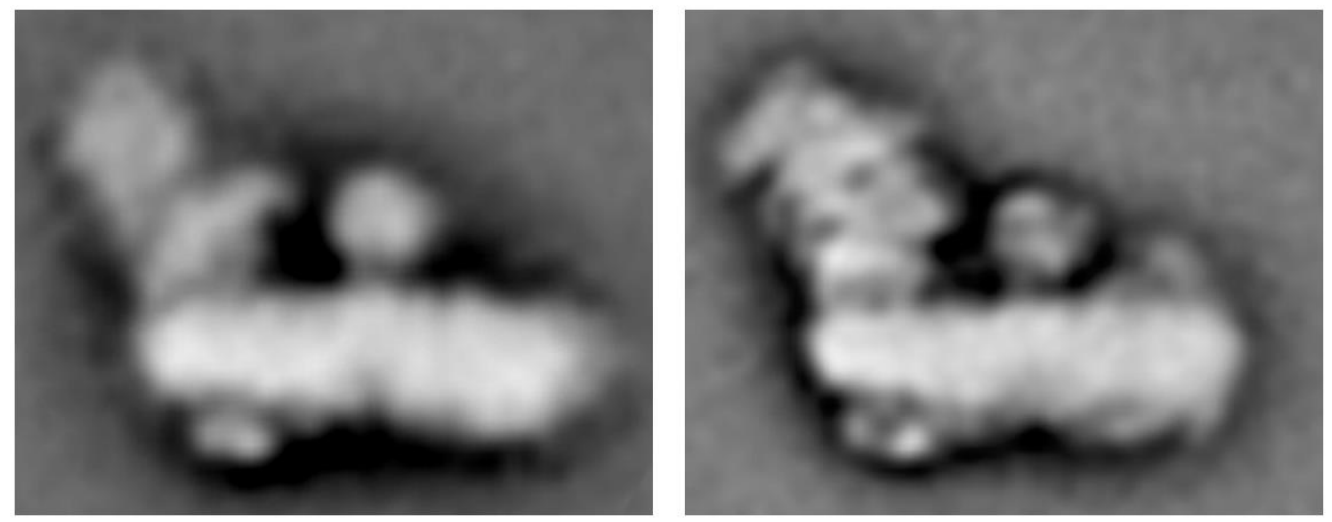

Figure 1: Structure of plant complex I as revealed by single particle negative stain electron microscopy. Left: Arabidopsis (Dudkina et al. 2015), right: potato (Bultema et al. 2009). 

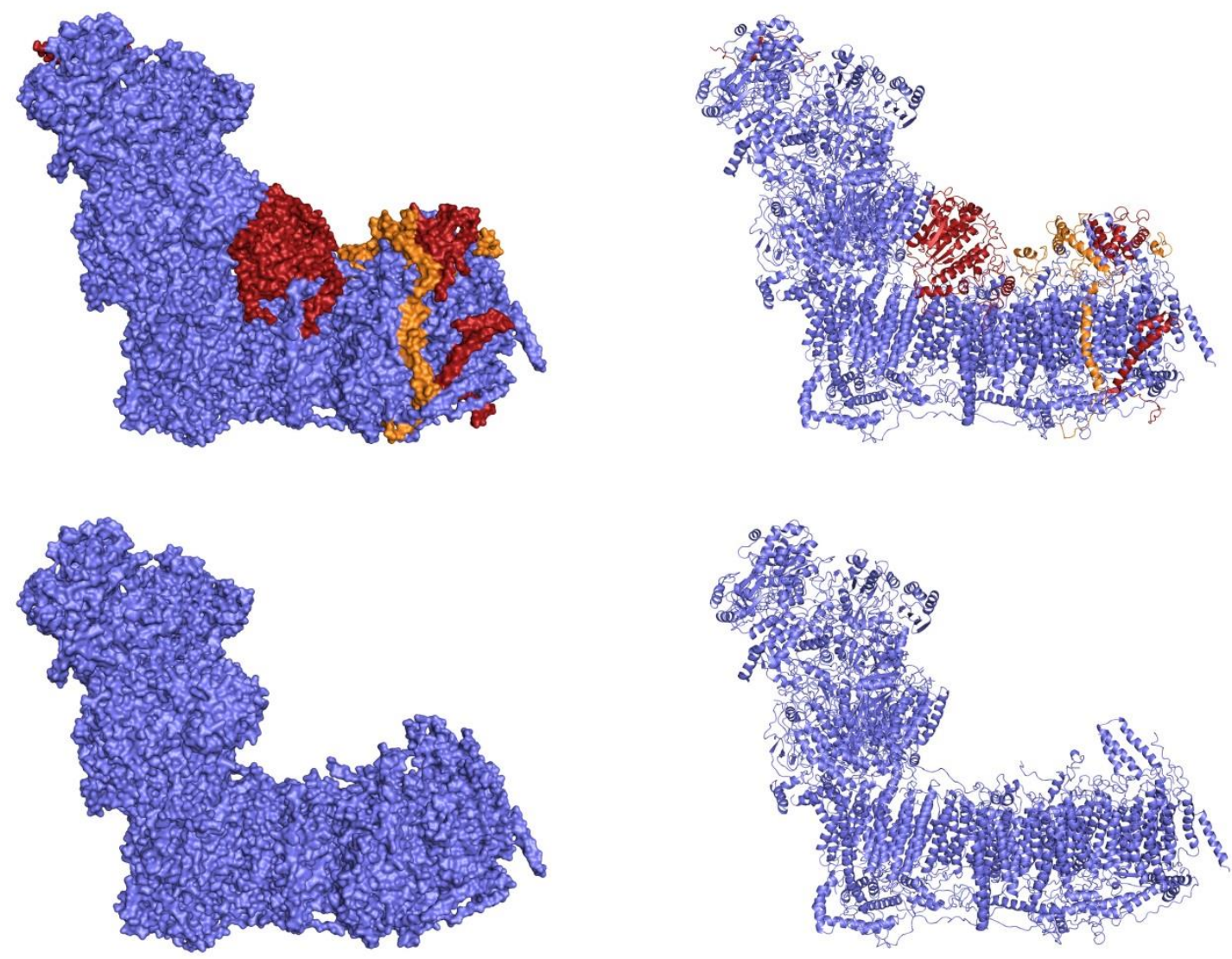

Figure 2: Locations of subunits of bovine complex I, which are absent in plants. The structure of bovine complex I was taken from Zhu et al. (2016) (Protein Data Bank accession: 5LDW). Structure processing and image generation was achieved using PyMol (https://www.pymol.org/). Bovine complex I includes 45 subunits, 40 of which are also present in Arabidopsis complex I. Red: Subunits absent in Arabidopsis (42 kDa, B17, $10 \mathrm{kDa}$, SDAP- $\alpha$, SDAP- $\beta$ ). Orange: Subunits lacking N-terminal portions (ASHI, B12, B15 and SGDH) or a C-terminal portion (B22) in Arabidopsis; see Appendix 2 for details. Top: complete structures of bovine complex I. Bottom: bovine complex I structures lacking subunits and $\mathrm{C}$ - or $\mathrm{N}$-terminal portions absent in Arabidopsis. Left: surface representation, right: ribbon representation. 

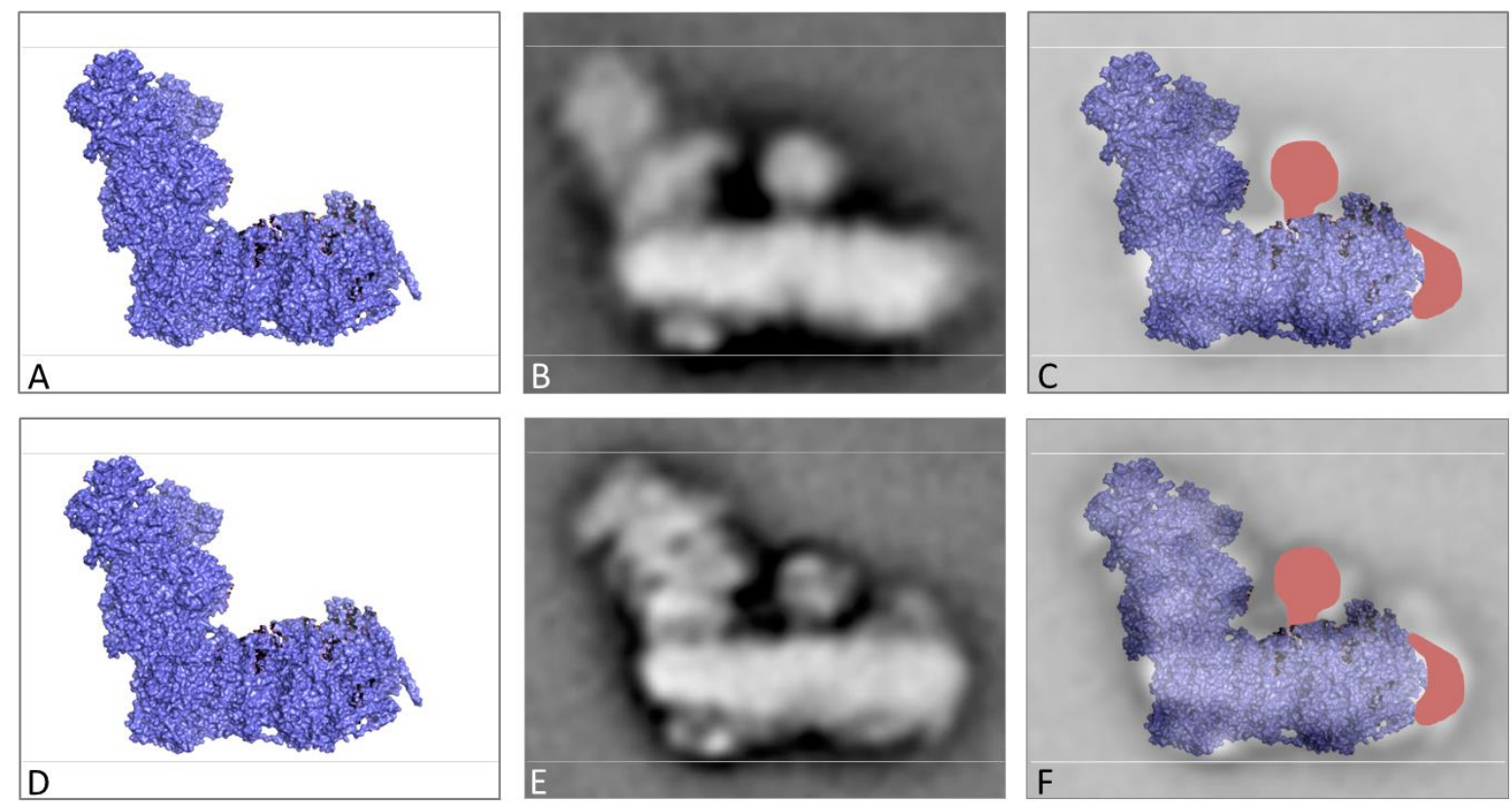

Figure 3: Comparison of complex I structures in mammals and plants. A, D: Structure of bovine complex I (Zhu et al. 2016) lacking five subunits absent in complex I from Arabidopsis thaliana and $\mathrm{C}$ - and $\mathrm{N}$-terminal portions of five additional subunits (see Fig. 2). B: Low resolution structure of Arabidopsis complex I as revealed by single particle negative stain electron microscopy (Dudkina et al. 2005). C: Superimposed structures of bovine complex I lacking subunits and portions of subunits absent in Arabidopsis and complex I from Arabidopsis. E: Low resolution structure of potato complex I as revealed by single particle negative stain electron microscopy (Bultema et al. 2009). F: Superimposed structures of bovine complex I lacking subunits and portions of subunits absent in Arabidopsis and complex I from potato. Extra mass in complex I from plants is indicated in red. 